\title{
Decreased microRNA-140-5p contributes to respiratory syncytial virus disease through targeting Toll-like receptor 4
}

\author{
YUN ZHANG $^{1}$ and LINGYUN SHAO ${ }^{2}$ \\ ${ }^{1}$ Infectious Disease Department, Jiangsu Taizhou People's Hospital, Taizhou, Jiangsu 225300; \\ ${ }^{2}$ Infectious Disease Department, Shanghai Huashan Hospital, Shanghai 200040, P.R. China
}

Received December 13, 2016; Accepted August 28, 2017

DOI: $10.3892 /$ etm.2018.6272

\begin{abstract}
The abnormal expression of miRNAs (miRs) has previously been reported in respiratory syncytial virus (RSV) disease. However, to the best of our knowledge, the expression of miR-140-5p in patients with an RSV infection has never been explored. Reverse transcription-quantitative polymerase chain reaction was performed to analyze the level of miR-140-5p in the blood and nasopharyngeal airway samples. ELISAs were performed to determine the levels of tumor necrosis factor $\alpha$, interleukin (IL)-1 $\beta$, IL-6 and IL-8. A dual luciferase reporter assay was also performed to investigate the possible target gene of miR-140-5p. The results demonstrated that the levels of miR-140-5p were significantly decreased in the nasal mucosal and peripheral blood samples of patients with RSV infection. It was also revealed that overexpression of miR-140-5p decreased the inflammatory responses, while inhibition of miR-140-5p enhanced the inflammatory responses. Additionally, three binding sites of miR-140-5p in the 3 'untranslated region (UTR) of Toll-like receptor (TLR) 4 were identified and a dual luciferase reporter assay demonstrated that miR-140-5p significantly suppressed the relative luciferase activity of pmirGLO-TLR4-3'UTR. Furthermore, the level of miR-140-5p was shown to be increased following interferon (IFN) $\alpha$ incubation. Notably, inhibition of miR-140-5p markedly attenuated IFN $\alpha$-mediated downregulation of tumor necrosis factor $\alpha$, and interleukin- $1 \beta,-6$ and -8 in BEAS-2B cells. In summary, decreased miR-140-5p levels are involved in RSV-infection diseases primarily through targeting TLR4.
\end{abstract}

\section{Introduction}

The mammalian Toll-like receptors (TLRs) serve key roles in innate immunity and can react to different microbes,

Correspondence to: Dr Yun Zhang, Infectious Disease Department, Jiangsu Taizhou People's Hospital, 399 Hailing South Road, Taizhou, Jiangsu 225300, P.R. China

E-mail: zhangyun160912@163.com

Key words: microRNA-140-5p, respiratory syncytial virus infection, Toll-like receptor 4 , interferon- $\alpha$ including gram-positive and -negative bacteria, mycobacteria and fungi (1-3). TLR2 was previously revealed to transduce signals from gram-positive bacteria and fungi (3-5), while TLR4 responded to bacterial lipopolysaccharide (LPS) (6). In addition, TLR4 was previously revealed to be a key regulator of the innate immune response following respiratory syncytial virus (RSV) infection $(7,8)$.

During winter epidemics the prevalence of RSV is higher than any other virus and RSV-associated infections are a common reason for hospitalization worldwide $(9,10)$. It has also been identified that RSV infections may develop into illnesses as mild as common colds to illnesses as serious as severe lower respiratory tract infections, and the transition between the mild and severe disease only requires $h(11,12)$. Thus, it is important for clinicians to effectively screen patients with an RSV infection.

MicroRNAs (miRs) are small non-coding RNA sequences that suppress the translation of mRNA through the incomplete base pairing mechanism $(13,14)$. Previous studies have demonstrated that miRs are widely involved in the innate and adaptive immune systems $(15,16)$. For instance, miR-221 was demonstrated to regulate RSV replication in the human bronchial epithelium through suppressing $\beta$-nerve growth factor expression (16). The primary focus of the present study was miR-140-5p, which was reported to be differentially expressed in numerous tumor types, including breast cancer, gastric cancer, biliary tract cancer $(13,14,17)$. However, to the best of our knowledge, the specific role of miR-140-5p in RSV infections has never been explored.

In the current study, the level of miR-140-5p was identified to be reduced in the nasopharyngeal airway (NPA) and peripheral blood samples of patients with an RSV infection compared with normal controls. Additional study revealed that TLR4 was a target gene of miR-140-5p, suggesting a potential therapeutic target in the treatment of patients with RSV infection.

\section{Materials and methods}

Study design. The heparinized blood and NPA samples were collected from patients with a bronchiole RSV infection, causing bronchiolitis, within $24 \mathrm{~h}$ of first contact with the hospital $(6.8 \pm 3.9$ years, $n=104 ; 45 \%$ male), and from healthy controls $(6.5 \pm 4.1$ years, $n=40 ; 55 \%$ male) during their annual physical examination, at Taizhou People's Hospital (Taizhou, 
China), between December 2015 and May 2016. The exclusion criteria were as previously described, including corticosteroid use $48 \mathrm{~h}$ prior to recruitment, serious congenital heart or lung disease and immunodeficiency, and the presence of $\geq 15$ viral pathogens (18). Based on the severity of the RSV infection, the patients were divided into three groups. The mild group consisted of those who were without hypoxia or severe feeding problems $(n=42)$. The moderate group consisted of those who required hospitalization to receive supplemental oxygen (oxygen saturation, <93\%) and/or nasogastric feeding ( $\mathrm{n}=34$ ). The severe group consisted of those who required mechanical ventilation $(n=28)$. Written informed consent was obtained from the parents of all children. Human studies were approved by the Taizhou People's Hospital Ethics Committee.

NPA samples for miRNA analysis. NPA samples were separated from nostrils by deep nasal suctioning. An RSV infection was determined using a rapid antigen test (Alere ${ }^{\mathrm{TM}}$ BinaxNOW ${ }^{\circledR}$ RSV Card; Alere, Inc., Waltham, MA, USA) according to the manufacturer's protocol and in-house reverse transcription-quantitative polymerase chain reaction (RT-qPCR) analysis.

Total RNA was isolated with RNAzol LS (Vigorous Biotechnology, Beijing, China) according to the specific instructions to isolate small RNAs. Quality, quantity and integrity of RNA were monitored using a NanoDrop spectrophotometer (ND-1,000, Nanodrop Technologies; Thermo Fisher Scientific, Inc., Waltham, MA, USA). The commercially available TaqMan ${ }^{\circledR}$ Fast Virus 1-Step Master mix (cat no. 4444432; Thermo Fisher Scientific, Inc.) was used to detect RSV. In brief, $5 \mu \mathrm{l}$ extracted nucleic acids was added in a reaction tube containing $5 \mu 1$ primer probe mixture and $10 \mu 1$ TaqMan $^{\circledR}$ Fast Virus 1-Step Master mix in order to perform cDNA synthesis and qPCR. Subsequently, the PCR amplification was performed using $1 \mathrm{mg}$ of cDNA and SYBR Green Master mix (Roche Diagnostics, Basel, Switzerland) on a Roche Lightcycler 480 (Roche Diagnostics). For qPCR, the mixture was incubated at $50^{\circ} \mathrm{C}$ for $10 \mathrm{~min}$. Denaturation followed at $95^{\circ} \mathrm{C}$ for $30 \mathrm{sec}$, then 10 cycles of PCR $(15 \mathrm{sec}$ at $95^{\circ} \mathrm{C}, 30 \mathrm{sec}$ at $53^{\circ} \mathrm{C}$ and $30 \mathrm{sec}$ at $60^{\circ} \mathrm{C}$ ) and 30 additional cycles of PCR for the detection of fluorescence signals (15 sec at $95^{\circ} \mathrm{C}, 30 \mathrm{sec}$ at $53^{\circ} \mathrm{C}$ and $30 \mathrm{sec}$ at $60^{\circ} \mathrm{C}$ ). Relative expression was normalized against the endogenous control, GAPDH, using the $2^{-\Delta \Delta C t}$ method (19). The primers used were as follows: RSV-A subtype-F: AGATCAACTTCTGTCATCCAGCAA; RSV-A-subtype-R: TTCTGCACATCATAATTAGGAG; RSV-B-subtype-F: AAGATGCAAATCATAAATTCACAG GA; RSV-B-subtype-R: TGATATCCAGCATCTTTAAGTA; GAPDH-F: GAGAAGGCTGGGGCTCATTT; GAPDH-R: AGTGATGGCATGGACTGTGG.

Then, the respiratory secretions, including phlegm and mucus, were removed from NPA samples by tracheal suction, and nasal epithelial cells were collected from each nostril by rotating a cytology brush (Ningbo Finer Medical Instruments Co., Ltd., Ningbo, China) over the anterior nasal mucosa. Following that, the brushes were immersed in the RNA stabilization reagent, RNAlater ${ }^{\circledR}$ (Sigma-Aldrich; Merck KGaA, Darmstadt, Germany). Epithelial cells were detached from the cytology brushes by placing the brush in a $15 \mathrm{ml}$ conical tube containing $8 \mathrm{ml}$ of DMEM (Hyclone; GE Healthcare
Life Sciences, Logan, UT, USA) and transported on ice. The tube was then centrifuged at $4^{\circ} \mathrm{C}$ at $400 \mathrm{x}$ g for $5 \mathrm{~min}$ and the supernatant was removed. Then, cells were stored at $-80^{\circ} \mathrm{C}$ in RNAlater for miRNA analysis.

ELISA. To evaluate the role of miR-140-5p in pro-inflammatory responses, miR-140-5p mimics or inhibitors were transfected into BEAS-2B cells for $48 \mathrm{~h}$ in the presence of $10 \mu \mathrm{g} / \mathrm{ml}$ LPS and the supernatant was collected for ELISA assay. In addition, BEAS-2B cells were also incubated with $0.1,0.5$ or $1.0 \mu \mathrm{g} / \mathrm{ml}$ IFN $\alpha$ (Sigma-Aldrich; Merck Millipore, Darmstadt, Germany) for $48 \mathrm{~h}$ and the supernatant was collected for further assay. Dimethyl sulfoxide (DMSO) was used as a control for IFN $\alpha$. BEAS-2B cells were treated in a lysis buffer $(50 \mathrm{mmol} / \mathrm{l}$ Tris-HCl, 300 mmol/1 NaCl, 5 mmol/l EDTA, $1 \%$ Triton X-100, $0.02 \%$ sodium azide) containing a protease inhibitor cocktail (Roche, Mannheim, Germany). The lysates were centrifuged at $16,000 \mathrm{x}$ g for $15 \mathrm{~min}$ at $4^{\circ} \mathrm{C}$ and supernatants were used to quantify the levels of TNF- $\alpha$ (cat no. DTA00C; Human TNF- $\alpha$ Quantikine ELISA kit), IL-6, (cat no. D6050; Human IL-6 Quantikine ELISA kit), IL-1 $\beta$ (cat no. DLB50; Human IL-1 beta/IL-1F2 Quantikine ELISA kit), and IL-8 (cat no. D8000C; Human IL-8/CXCL8 Quantikine ELISA kit) by way of a sandwich ELISA following the manufacturers' protocols (R\&D Systems, Minneapolis, Minnesota, USA). Samples were read at a $450 \mathrm{~nm}$ wavelength using a microplate reader (Model 3550; Thermo Fisher Scientific, Inc.).

Cell culture. Human bronchial epithelial cells, BEAS-2B were purchased from the American Type Culture Collection (Manassas, VA, USA) and cultured in Dulbecco's modified Eagle's medium (DMEM) (Hyclone; GE Healthcare Life Sciences) supplemented with $10 \%(\mathrm{v} / \mathrm{v})$ horse serum (Hyclone; GE Healthcare Life Sciences), $100 \mathrm{U} / \mathrm{ml}$ penicillin (Invitrogen; Thermo Fisher Scientific, Inc.) and $0.1 \mathrm{mg} / \mathrm{ml}$ streptomycin (Hyclone; GE Healthcare Life Sciences) at $37^{\circ} \mathrm{C}$ in a humidified atmosphere with $5 \% \mathrm{CO}_{2}$.

RNA extraction. Total RNA (10 $\mu \mathrm{g})$ from the whole blood samples ( $5 \mathrm{ml})$, in tubes containing EDTA, or BEAS-2B cells was isolated using RNAVzol LS (Vigorous Biotechnology Beijing Co., Ltd., Beijing, China) in accordance with the manufacturer's protocol. The concentration and purity of the RNA samples were determined by the OD260/OD280 ratio using a microplate reader (Model 3550; Thermo Fisher Scientific, Inc.).

RT-qPCR analysis. For synthesis of cDNA of the specific $\mathrm{miR}, 1 \mu \mathrm{g}$ of the total RNA was reverse transcribed using TaqMan $^{\text {TM }}$ MicroRNA Reverse Transcription kit (Applied Biosystems; Thermo Fisher Scientific, Inc.) with specific primers for miR-140-5p and U6 (Shanghai Sangon Technology Co., Ltd., Shanghai, China). To quantify the miR-140-5p, a qPCR assay was performed using $\mathrm{iQ}^{\mathrm{TM}} \mathrm{SYBR}^{\circledR}$ Green Supermix (Bio-Rad Laboratories, Inc., Hercules, CA, USA) in an iCycler $\mathrm{iQ}^{\mathrm{TM}}$ qPCR detection system (both Bio-Rad Laboratories, Inc). The PCR amplifications were performed in a $10 \mu \mathrm{l}$ reaction system containing $5 \mu \mathrm{l}$ SYBR-Green Supermix, $0.4 \mu \mathrm{l}$ forward primer, $0.4 \mu \mathrm{l}$ reverse primer, $2.2 \mu \mathrm{l}$ double distilled $\mathrm{H}_{2} \mathrm{O}$ and $2 \mu \mathrm{l}$ template cDNA. The thermal cycling conditions were as follows: A hot start step at $95^{\circ} \mathrm{C}$ for 
$10 \mathrm{~min}$, followed by 40 cycles of $95^{\circ} \mathrm{C}$ for $15 \mathrm{sec}$ and $60^{\circ} \mathrm{C}$ for $1 \mathrm{~min}$, annealing at $55^{\circ} \mathrm{C}$ for $30 \mathrm{sec}$ and elongation at $72^{\circ} \mathrm{C}$ for $3 \mathrm{~min}$. The relative level of $\mathrm{miR}-140-5 \mathrm{p}$ was determined using the $2^{-\Delta \Delta \mathrm{Cq}}$ analysis method (19). U6 was selected as the internal control. The primers used in the present study were as follows: miR-140-5p-RT, 5'-GTCGTATCCAGTGCAGGGTCCGAG GTATTCGCACTGGATACGACCTACCA-3'; U6-RT, 5'-GTC GTATCCAGTGCAGGGTCCGAGGTATTCGCACTGGAT ACGACAAAATG-3' miR-140-5p, forward, 5'-GCGCGC AGUGGUUUUACCCUA-3'; U6, forward, 5'-GCGCGTCGT GAAGCGTTC-3'; universal reverse primer, 5'-GTGCAGGGT CCGAGGT-3'. RT stands for stem loop primer here.

Transient transfection. Firstly, $6 \times 10^{5}$ cells were equally seeded in the 6-well plates with $2 \mathrm{ml}$ DMEM culture medium containing serum and antibiotics. miR-140-5p mimics, inhibitors or miR negative controls were purchased from GenePharma (Shanghai, China). Simultaneously, miR-140-5p mimics, inhibitors or miR negative controls were mixed with HiperFect transfection reagent (Qiagen $\mathrm{GmbH}$, Hilden, Germany) and incubated at room temperature for $10 \mathrm{~min}$. Then, each complex was transfected into two wells containing the BEAS-2B cells for $48 \mathrm{~h}$ at a final concentration of $10 \mathrm{nM}$. The specific sequences were as follows: miR-140-5p mimic, CAGUGGUUUUACCCUAUGGUAG; miR-140-5p inhibitor: CUACCAUAGGGUAAAACCACUG; miR NC for mimic: UUCUCCGAACGUGUCAACGUTT; miR NC for inhibitor: CAGUACUUUGUGUAGUACAA.

Bioinformatics analysis. TargetScan 7.1 (www.targetscan. org/vert_71/) was applied to predict the possible target genes of $\mathrm{miR}-140-5 \mathrm{p}$.

Dual luciferase reporter assay. The 3'untranslated region (UTR) of TLR4, which contains the predicted target site for miR-140-5p, was cloned into the pmirGLO luciferase reporter vector (Promega Corporation, Madison, WI, USA), which was cleaved at $\mathrm{SacI}$ and $\mathrm{XhoI}$ sites. The two different 3'UTR regions containing the potential binding sites of TLR4 were cloned into the luciferase reporter system, which were designated pmirGLO-TLR4-3'UTR-1 and pmirGLO-TLR4-3'UTR-2. The former contained the first binding site, while the latter included the second and the third binding sites. Details of PCR procedures are described as follows: A hot start step at $95^{\circ} \mathrm{C}$ for $10 \mathrm{~min}$, followed by 40 cycles of $95^{\circ} \mathrm{C}$ for $15 \mathrm{sec}, 55^{\circ} \mathrm{C}$ for $45 \mathrm{sec}$ and $72^{\circ} \mathrm{C}$ for $30 \mathrm{sec}$.

Prior to conducting the dual reporter assay, $5 \times 10^{4}$ BEAS- $2 B$ cells/well were seeded in 24-well plates with $500 \mu 1$ DMEM medium and cultured for $18 \mathrm{~h}$. The cells were transfected with the modified firefly luciferase reporter vector $(500 \mathrm{ng} / \mu \mathrm{l})$ mixed with Vigofect transfection reagent (Vigorous, Beijing, China) according to the manufacturer's protocol. After continuous exposure of miR-140-5p (CAGUGGUUUUACCCUAUG GUAG)/pmirGLO-TLR4-3'UTR-1 or pmirGLO-TLR4-3'UTR-2 or NC (UUCUCCGAACGUGUCAACGUTT)/pmirGLO blank vector for $48 \mathrm{~h}$, the luciferase activities of firefly and Renilla were measured with the Dual-Luciferase ${ }^{\circledR}$ Reporter Assay system (Promega Corporation) according to the manufacturer's protocol. Firefly luciferase activity was normalized to Renilla luciferase activity.
Western blotting. Cell protein was extracted using radioimmunoprecipitation lysis buffer (Beijing Solarbio Science \& Technology Co., Ltd., Beijing, China) and was collected following centrifugation at $12,000 \mathrm{x}$ g for $30 \mathrm{~min}$ at $4^{\circ} \mathrm{C}$. A bicinchoninic protein assay kit (Pierce; Thermo Fisher Scientific, Inc.) was used to determine the protein concentration. A total of $15 \mu \mathrm{g}$ protein was loaded per lane, separated by $10 \%$ SDS-PAGE and transferred to polyvinylidene difluoride membranes. The membranes were blocked with $8 \%$ non-fat dry milk at $4^{\circ} \mathrm{C}$ overnight. Following three washes with PBS with Tween 20 (5 $\mathrm{min} /$ wash), the membranes were incubated with the following primary antibodies at $4^{\circ} \mathrm{C}$ overnight: TLR4 (cat no. 14358; 1:1,000; Cell Signaling Technology, Inc., Danvers, MA, USA) and GAPDH (cat. no. 5174; 1:1,000; Cell Signaling Technology, Inc.). Following several washes with TBST, the membranes were incubated with horseradish-peroxidase (HRP)-conjugated goat anti-rabbit and anti-mouse Immunoglobulin $\mathrm{G}$ (IgG) or HRP-conjugated mouse anti-goat IgG (all 1:5,000; Zhongshan Gold Bridge Biological Technology Co., Beijing, China) for $2 \mathrm{~h}$ at room temperature and then washed. The blots were then incubated with horseradish peroxidase (HRP)-conjugated anti-IgG secondary antibody (1:5,000; OriGene Technologies, Inc., Beijing, China) for $2 \mathrm{~h}$ at room temperature and then washed followed by detection with enhanced chemiluminescent substrate (EMD Millipore, Billerica, MA, USA). GAPDH was used as an internal control. ImageJ software (National Institutes of Health, Bethesda, MD, USA) was used for density analysis.

Interferon- $\alpha$ (IFN $\alpha$ ) treatment. In brief, BEAS-2B cells were seeded at a density of $5 \times 10^{5}$ cells/well in a 6 -well plate for $24 \mathrm{~h}$. Then, BEAS-2B cells were incubated with $0.1,0.5$ or $1.0 \mu \mathrm{g} / \mathrm{ml}$ Recombinant Human IFN $\alpha$ (CYT-283, ProSpec, Rehovot, Israel) or the corresponding volume of distilled water (control) for $48 \mathrm{~h}$. Then, the RNA was isolated and the level of miR-140-5p was quantified using RT-qPCR according to the aforementioned method. The primers used were as follows: MiR-140-5p-Stem: GTCGTATCCAGTGCAGGGTCCGAG GTATTCGCACTGGATACGACCTACCA; U6-Stem: GTC GTATCCAGTGCAGGGTCCGAGGTATTCGCACTGGAT ACGACAAATATG; miR-140-5p-F: GCCAGTGGTTTT ACCCTAT; U6-F: GCGCGTCGTGAAGCGTTC; universal reverse: GTGCAGGGTCCGAGGT.

Statistical analysis. Data are presented as mean \pm standard deviation. To compare two groups, the two-tailed unpaired Student's t test was performed. For multiple groups comparisons, one-way analysis of variance followed by Tukey's post hoc test was used. Statistical tests were performed using SPSS software (version 13.0; SPSS, Inc., Chicago, IL, USA). P<0.05 was considered to indicate a statistically significant difference.

\section{Results}

miR-140-5p levels decrease in the NPAs and peripheral blood of patients with an RSV infection. The clinical characteristics of patients with an RSV infection are demonstrated in Table I. To determine the level of miR-140-5p in the NPA samples, the RNA was extracted from the patients with RSV infections and the healthy controls. Compared with that of the healthy controls, miR-140-5p expression was significantly lower in 
Table I. Clinicopathological characteristics of controls and patients with RSV included in the reverse transcription-quantitative polymerase chain reaction analysis.

\begin{tabular}{lcccc}
\hline & & \multicolumn{3}{c}{ Severity of RSV } \\
\cline { 3 - 4 } Characteristic & Control & Mild & Moderate & Severe \\
\hline $\mathrm{n}$ & 40 & 42 & 34 & 28 \\
Age, years & $6.5(4.1)$ & $4.6(3.8)$ & $5.8(3.7)$ & $5.2(3.9)$ \\
Male, $\mathrm{n}(\%)$ & $22(55)$ & $18(43)$ & $16(47)$ & $13(46)$ \\
Weight, $\mathrm{g}$ & $5,873 \pm 1,647$ & $6,998 \pm 2,350$ & $6,435 \pm 1,392$ & $5,967 \pm 1,845$ \\
Duration of symptoms, days & & $4.6(3-6)$ & $4.8(4-5)$ & $4.3(3-5)$ \\
Admitted to a ward, $\mathrm{n}(\%)$ & $6(14)$ & $34(100)$ & $28(100)$ \\
Length of stay, days & & $0(0-0)$ & $3(1-4)$ & $4(2-6)$ \\
Length of stay >3 days, $\mathrm{n}(\%)$ & $0(0)$ & $7(21)$ & $16(57)$ \\
\hline
\end{tabular}

Data are presented as the mean \pm standard deviation or median (range). n, number; RSV, respiratory syncytial virus.

patients with RSV infections (Fig. 1A). The lowest miR-140-5p level in the NPA samples was exhibited by patients with severe RSV infections, followed by patients with moderate and mild infections, which were significantly lower compared with the controls. Furthermore, the level of miR-140-5p in the peripheral blood samples of patients with RSV infections and healthy control was analyzed. Compared with the healthy controls, the level of miR-140-5p was significantly decreased in patients with RSV infections, with the biggest decrease in severe $\mathrm{RSV}$, followed by the moderate and mild disease subgroups (Fig. 1B).

Decreased miR-140-5p expression levels induce pro-inflammatory responses. The overexpression of $\mathrm{miR}-140-5 \mathrm{p}$ significantly decreased the levels of pro-inflammatory factors, tumor necrosis factor (TNF) $\alpha$ (Fig. 2A), interleukin (IL)-1 $\beta$ (Fig. 2B), IL-6 (Fig. 2C) and IL-8 (Fig. 2D). By contrast, inhibition of miR-140-5p significantly enhanced the production of TNF $\alpha$ (Fig. 2E), IL-1 $\beta$ (Fig. 2F), IL-6 (Fig. 2G) and IL-8 (Fig. 2H).

TLR4 may be a direct target of miR-140-5p. The mechanism underlying the involvement of miR-140-5p in RSV infections was explored. Bioinformatic analysis (targetscan.org/vert_71/) demonstrated that one conserved and two poorly conserved (binding site 1 was conserved, while binding site 2 and 3 were poorly conserved) binding sites were identified in the 3'UTR of TLR4 (Fig. 3A). The dual luciferase reporter assay demonstrated that miR-140-5p significantly suppressed the relative luciferase activity of pmirGLO-TLR4-3'UTR-1 and pmirGLO-TLR4-3'UTR-2 (Fig. 3B), suggesting that TLR4 is a direct target gene of miR-140-5p. In addition, the overexpression of miR-140-5p significantly suppressed the protein level of TLR4 in BEAS-2B cells (Fig. 3C), while inhibition of miR-140-5p significantly enhanced the expression of TLR4 in BEAS-2B cells (Fig. 3D).

IFN $\alpha$ treatment increases the level of miR-140-5p. BEAS-2B cells were incubated with $0.1,0.5$ or $1.0 \mu \mathrm{g} / \mathrm{ml}$ IFN $\alpha$ for $48 \mathrm{~h}$ and the level of miR-140-5p was demonstrated to increase in a dose-dependent manner following IFN $\alpha$ incubation (Fig. 4A). miR-140-5p levels significantly increased with 0.5 and $1.0 \mu \mathrm{g} / \mathrm{ml} \mathrm{IFN} \alpha$ compared with the control. In addition, the supernatant of the BEAS-2B cells incubated with IFN $\alpha$ was collected for ELISA assay. Following the incubation of $1.0 \mu \mathrm{g} / \mathrm{ml} \mathrm{IFN} \alpha$, the production of TNF $\alpha$ (Fig. 4B), IL-1 $\beta$ (Fig. 4C), IL-6 (Fig. 4D) and IL-8 (Fig. 4E) in BEAS-2B cells was significantly suppressed compared with the controls. By contrast, inhibition of miR-140-5p significantly attenuated the IFN $\alpha$-mediated downregulation of TNF $\alpha$, IL-1 $\beta$, IL- 6 and IL- 8 in BEAS-2B cells compared with the IFN $\alpha$-treated cells (Fig. 4B-E).

\section{Discussion}

The abnormal expression of miRNAs in different diseases has been widely reported $(15,16,20)$. For instance, studies have demonstrated that miR-221, let-7i and miR-30b regulate RSV replication in the human bronchial epithelium $(16,21)$. In the current study, the expression of miR-140-5p in the NPA and whole peripheral blood samples of patients with acute RSV disease was explored. Compared with the healthy controls, miR-140-5p was lowest in the NPA samples and peripheral blood of patients with a severe RSV infection, followed by patients with moderate and mild RSV infections. These in vivo results indicated that miR-140-5p may function as a potential biomarker for RSV infections and that there is a negative association between miR-140-5p levels and the severity of RSV infections. Notably, the overexpression and inhibition of miR-140-5p decreased and increased the levels of inflammation factors, respectively. The in vitro results from the present study revealed the anti-inflammatory effects of miR-140-5p, suggesting that inhibition of miR-140-5p contributes to inflammatory responses and that it may possibly serve a protective role in preventing RSV infections.

An RSV infection primarily affects the epithelial cells of the respiratory mucosa (22). By binding to TLR4, RSV triggers the abnormal activation of the nuclear factor- $\kappa \mathrm{B}$ signaling pathway $(23,24)$. Thus far, the regulation of TLR4 following RSV infection remains unknown. The present 
A

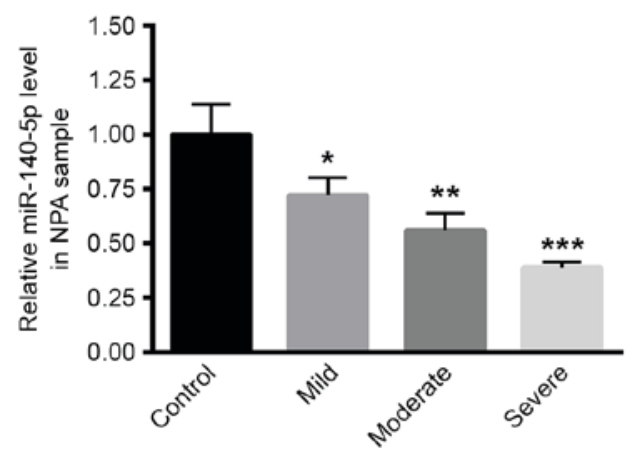

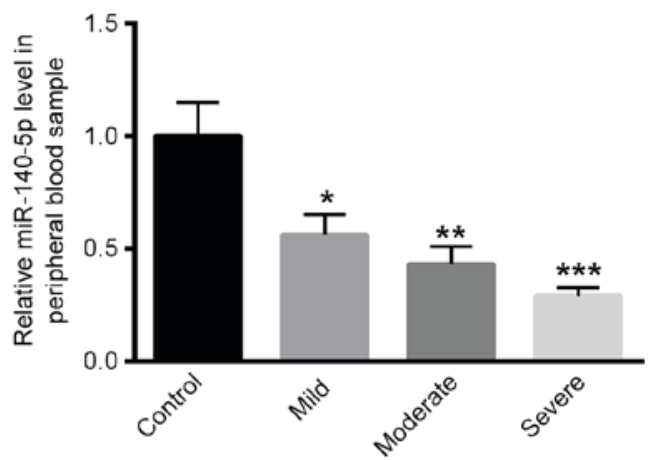

Figure 1. miR-140-5p levels decrease in the NPA and peripheral blood of patients with respiratory syncytial virus infections. Reverse transcription-quantitative polymerase chain reaction was performed to analyze miR-140-5p levels in (A) NPA and (B) peripheral blood samples. ${ }^{*} \mathrm{P}<0.05,{ }^{* *} \mathrm{P}<0.01,{ }^{* * * *} \mathrm{P}<0.001 \mathrm{vs}$. control. NPA, nasopharyngeal airway; miR, microRNA.

A

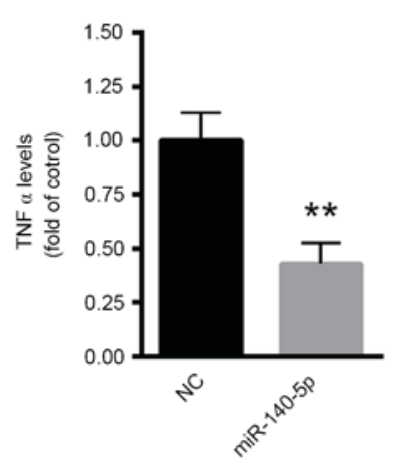

$E$

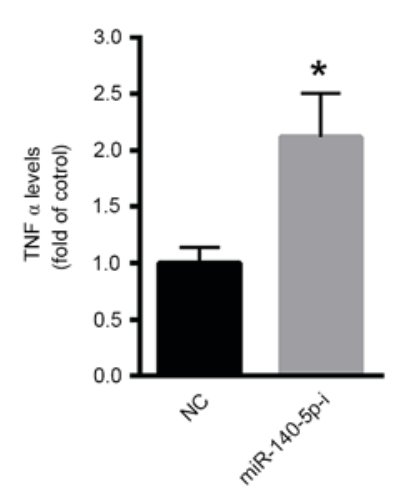

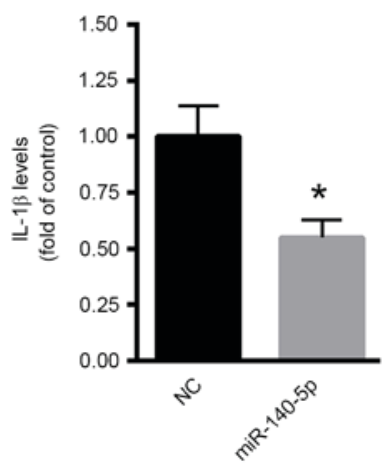

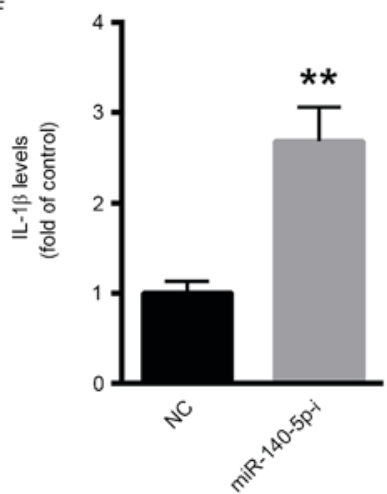

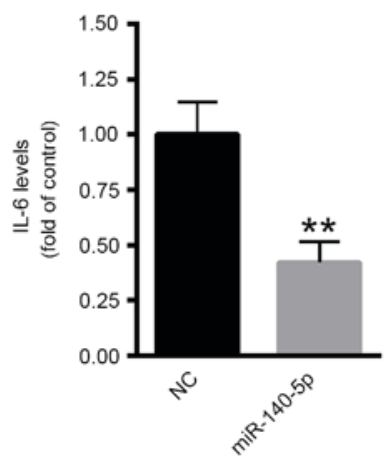

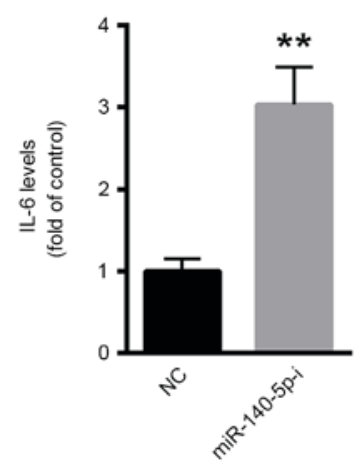

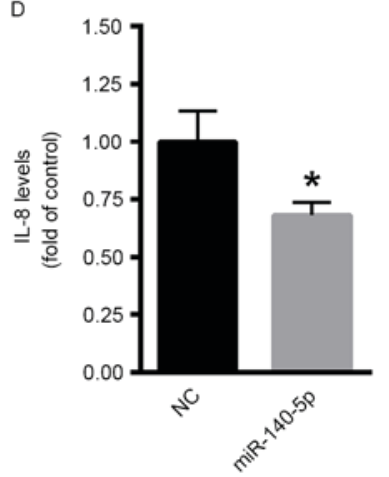

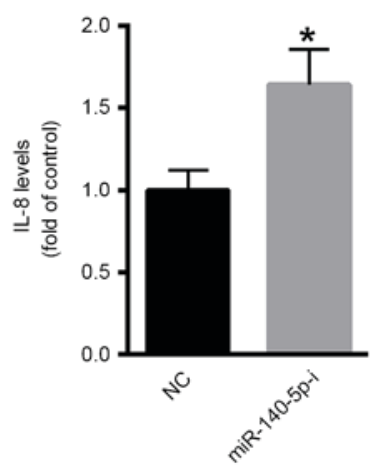

Figure 2. Decreased miR-140-5p expression levels induce pro-inflammatory responses. ELISA analysis of (A and E) TNF $\alpha$, (B and F) IL-1 $\beta$, (C and G) IL-6 and (D and H) IL-8 production in the BEAS-2B cells transfected with miR-140-5p mimic or inhibitor. ${ }^{*} \mathrm{P}<0.05,{ }^{* *} \mathrm{P}<0.01 \mathrm{vs}$. control. NC, negative control; miR, microRNA; miR-140-5p-i, miR-140-5p inhibitor; TNF, tumor necrosis factor; IL, interleukin.

study demonstrates that TLR4 may be a target gene of miR-140-5p. In vitro investigations revealed that decreased miR-140-5p levels in the NPA samples contributed to the immune response to RSV, this may primarily be due to the targeting of TLR4 by miR-140-5p.

Upon RSV infection, type I IFNs are secreted through the IFN $\alpha / \beta$ receptor to stimulate the expression of key proteins that modulate the process of viral replication and immune responses $(25,26)$. Then, IFNs trigger cell intrinsic antiviral responses, stimulate natural killer cells, macrophages and dendritic cells, and modulate the innate and adaptive immune responses $(25,27)$. IFN $\alpha / \beta$ expression is induced in bronchial epithelial cells following the infection of various viruses, including the influenza virus, RSV and human metapneumovirus $(26,28,29)$. To investigate whether miR-140-5p was involved in IFN $\alpha$-mediated antiviral therapy in the airways, the expression of miR-140-5p in BEAS-2B cells treated with IFN $\alpha$ was investigated. It was demonstrated that treatment with 0.5 or $1.0 \mu \mathrm{g} / \mathrm{ml}$ IFN $\alpha$ significantly increased miR-140-5p levels in BEAS-2B cells. Notably, the suppression of miR-140-5p significantly attenuated the IFN $\alpha$-mediated downregulation of TNF $\alpha$, IL-1 $\beta$, IL- 6 and IL- 8 in BEAS-2B cells. These 


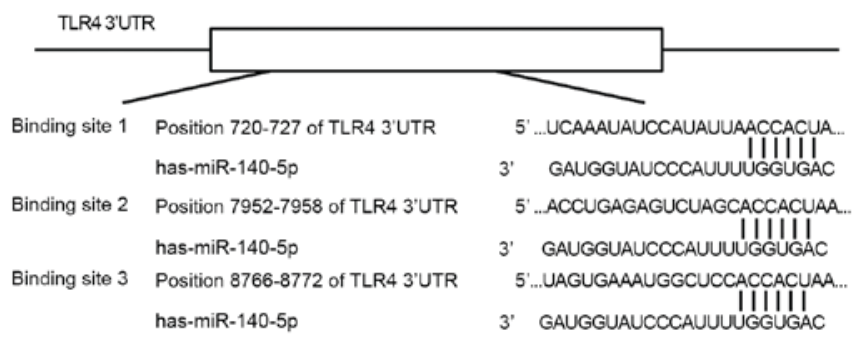

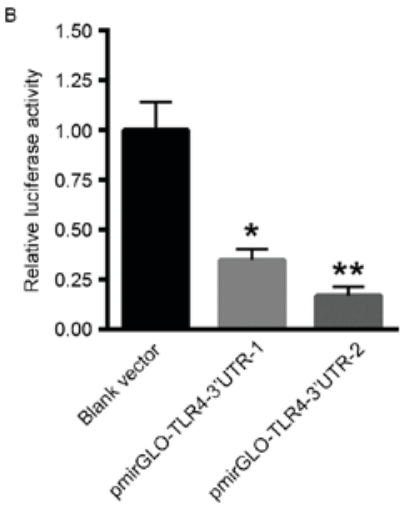

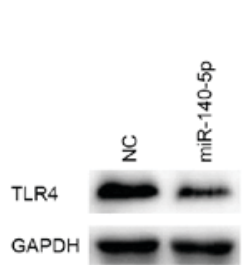

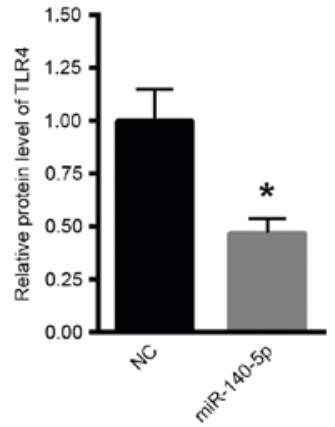

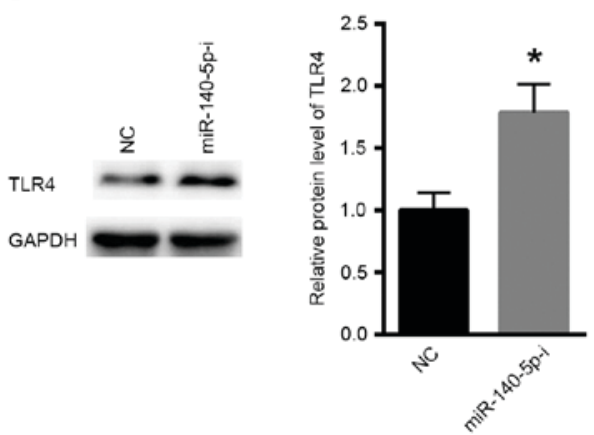

Figure 3. TLR4 is a direct target of miR-140-5p. (A) Schematic demonstration of the potential binding sites (Binding site 1 was conserved, while binding site 2 and 3 were poorly conserved) for miR-140-5p in the 3'UTR of TLR4. (B) Dual luciferase reporter assay. pmirGLO-TLR4-3'UTR-1 contained binding site 1 and pmirGLO-TLR4-3'UTR-2 contained binding sites 2 and 3. TLR4 protein expression due to the (C) overexpression and (D) inhibition of miR-140-5p was analyzed in BEAS-2B cells. ${ }^{*} \mathrm{P}<0.05,{ }^{* *} \mathrm{P}<0.01$ vs. control. NC, negative control; miR, microRNA; miR-140-5p-i, miR-140-5p inhibitor; TLR, Toll-like receptor; UTR, untranslated region.

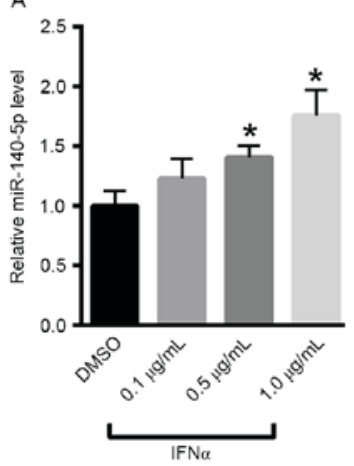

D

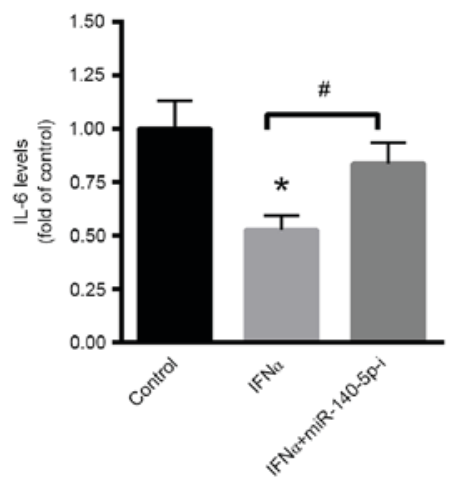

B

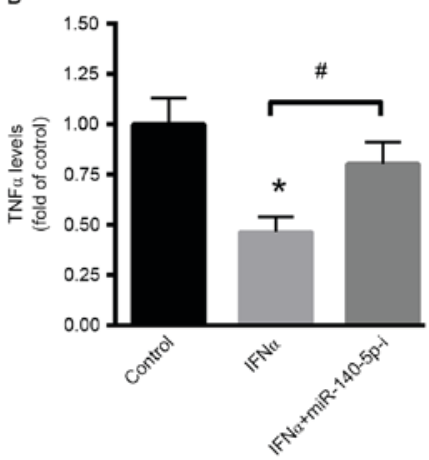

c

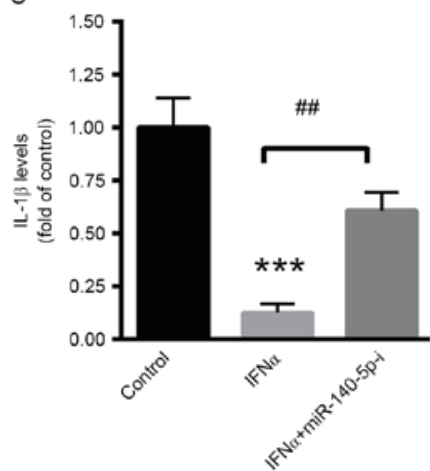

$\mathrm{E}$

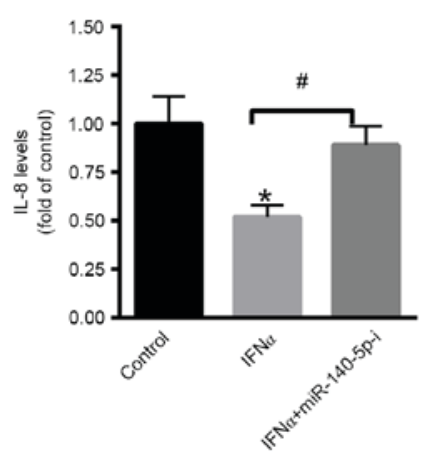

Figure 4. INF $\alpha$ treatment increased the level of miR-140-5p and decreased the levels of TNF $\alpha$, IL-1 $\beta$, IL-6 and IL-8. (A) Quantitative polymerase chain reaction was performed to analyze miR-140-5p levels following IFN $\alpha$ incubation. Stimulation of IFNa significantly suppressed the production of (B) TNF $\alpha$, (C) IL-1 $\beta$, (D) IL-6 and (E) IL-8 in BEAS-2B cells. "P<0.05, ${ }^{* * *} \mathrm{P}<0.001$ vs. control; ${ }^{*} \mathrm{P}<0.05,{ }^{\# \#} \mathrm{P}<0.01$ as indicated. INF, interferon; DMSO, dimethyl sulfoxide; $\mathrm{TNF}$, tumor necrosis factor; IL, interleukin; miR, microRNA; miR-140-5p-i, miR-140-5p inhibitor. 
data suggest that miR-140-5p is involved in INF $\alpha$-mediated anti-RSV infection therapy.

In conclusion, the current study demonstrated that miR-140-5p was reduced in the NPA and peripheral blood samples of patients with RSV infections compared with that of the healthy controls. Also, inhibiting miR-140-5p enhanced the pro-inflammatory responses, primarily by targeting TLR4. IFN $\alpha$ treatment was demonstrated to enhance the level of miR-140-5p in BEAS-2B cells. Thus, miR-140-5p may be a potential therapeutic target in patients with RSV infection.

\section{References}

1. Hirschfeld M, Kirschning CJ, Schwandner R, Wesche H, Weis JH, Wooten RM and Weis JJ: Cutting edge: Inflammatory signaling by Borrelia burgdorferi lipoproteins is mediated by toll-like receptor 2. J Immunol 163: 2382-2386, 1999.

2. Means TK, Wang S, Lien E, Yoshimura A, Golenbock DT and Fenton MJ: Human toll-like receptors mediate cellular activation by Mycobacterium tuberculosis. J Immunol 163: 3920-3927, 1999.

3. Schwandner R, Dziarski $R$, Wesche $H$, Rothe $M$ and Kirschning CJ: Peptidoglycan- and lipoteichoic acid-induced cell activation is mediated by toll-like receptor 2. J Biol Chem 274 17406-17409, 1999.

4. Takeuchi O, Hoshino K, Kawai T, Sanjo H, Takada H, Ogawa T, Takeda K and Akira S: Differential roles of TLR2 and TLR4 in recognition of gram-negative and gram-positive bacterial cell wall components. Immunity 11: 443-451, 1999.

5. Hua F, Ma J, Ha T, Kelley JL, Kao RL, Schweitzer JB, Kalbfleisch JH, Williams DL and Li C: Differential roles of TLR2 and TLR4 in acute focal cerebral ischemia/reperfusion injury in mice. Brain Res 1262: 100-108, 2009.

6. Ingalls RR, Lien E and Golenbock DT: Differential roles of TLR2 and TLR4 in the host response to Gram-negative bacteria: Lessons from a lipopolysaccharide-deficient mutant of Neisseria meningitidis. J Endotoxin Res 6: 411-415, 2000.

7. Underhill DM and Gantner B: Integration of Toll-like receptor and phagocytic signaling for tailored immunity. Microbes Infect 6: 1368-1373, 2004.

8. Underhill DM, Ozinsky A, Smith KD and Aderem A: Toll-like receptor-2 mediates mycobacteria-induced proinflammatory signaling in macrophages. Proc Natl Acad Sci USA 96: 14459-14463, 1999.

9. Henrickson KJ, Hoover S, Kehl KS and Hua W: National disease burden of respiratory viruses detected in children by polymerase chain reaction. Pediatr Infect Dis J 23 (1 Suppl): S11-S18, 2004.

10. Black CP: Systematic review of the biology and medical management of respiratory syncytial virus infection. Respir Care 48: 209-233, 2003.

11. Berger TM, Aebi C, Duppenthaler A and Stocker M; Swiss Pediatric Surveillance Unit: Prospective population-based study of RSV-related intermediate care and intensive care unit admissions in Switzerland over a 4-year period (2001-2005). Infection 37: 109-116, 2009.

12. Mansbach JM, Clark S, Christopher NC, LoVecchio F, Kunz S, Acholonu U and Camargo CA Jr: Prospective multicenter study of bronchiolitis: Predicting safe discharges from the emergency department. Pediatrics 121: 680-688, 2008.
13. Salem O, Erdem N, Jung J, Münstermann E, Wörner A, Wilhelm H, Wiemann S and Körner C: The highly expressed 5'isomiR of hsa-miR-140-3p contributes to the tumor-suppressive effects of miR-140 by reducing breast cancer proliferation and migration. BMC Genomics 17: 566, 2016.

14. Zou J and Xu Y: MicroRNA-140 inhibits cell proliferation in gastric cancer cell line HGC-27 by suppressing SOX4. Med Sci Monit 22: 2243-2252, 2016

15. Bakre A, Mitchell P, Coleman JK, Jones LP, Saavedra G, Teng M, Tompkins SM and Tripp RA: Respiratory syncytial virus modifies microRNAs regulating host genes that affect virus replication. J Gen Virol 93: 2346-2356, 2012.

16. Othumpangat S, Walton C and Piedimonte G: MicroRNA-221 modulates RSV replication in human bronchial epithelium by targeting NGF expression. PLoS One 7: e30030, 2012.

17. Yu J, Zhang W, Tang H, Qian H, Yang J, Zhu Z, Ren P and Lu B Septin 2 accelerates the progression of biliary tract cancer and is negatively regulated by mir-140-5p. Gene 589: 20-26, 2016.

18. Templeton KE, Scheltinga SA, Beersma MF, Kroes AC and Claas EC: Rapid and sensitive method using multiplex real-time PCR for diagnosis of infections by influenza a and influenza B viruses, respiratory syncytial virus, and parainfluenza viruses 1 , 2, 3, and 4. J Clin Microbiol 42: 1564-1569, 2004.

19. Livak KJ and Schmittgen TD: Analysis of relative gene expression data using real-time quantitative PCR and the 2(-Delta Delta C(T)) method. Methods 25: 402-408, 2001.

20. Mi QS, Xu YP, Wang H, Qi RQ, Dong Z and Zhou L: Deletion of microRNA miR-223 increases Langerhans cell cross-presentation. Int J Biochem Cell Biol 45: 395-400, 2013.

21. Thornburg NJ, Hayward SL and Crowe JE Jr: Respiratory syncytial virus regulates human microRNAs by using mechanisms involving beta interferon and NF-kB. MBio 3: pii: e00220-12, 2012.

22. Schröder NW and Arditi M: The role of innate immunity in the pathogenesis of asthma: Evidence for the involvement of toll-like receptor signaling. J Endotoxin Res 13: 305-312, 2007.

23. Abe T, Hemmi H, Miyamoto H, Moriishi K, Tamura S, Takaku H, Akira S and Matsuura Y: Involvement of the toll-like receptor 9 signaling pathway in the induction of innate immunity by baculovirus. J Virol 79: 2847-2858, 2005.

24. Haynes LM, Moore DD, Kurt-Jones EA, Finberg RW, Anderson LJ and Tripp RA: Involvement of toll-like receptor 4 in innate immunity to respiratory syncytial virus. J Virol 75: 10730-10737, 2001.

25. Randall RE and Goodbourn S: Interferons and viruses: An interplay between induction, signalling, antiviral responses and virus countermeasures. J Gen Virol 89: 1-47, 2008.

26. Durbin RK, Kotenko SV and Durbin JE: Interferon induction and function at the mucosal surface. Immunol Rev 255: 25-39, 2013.

27. González-Navajas JM, Lee J, David M and Raz E: Immunomodulatory functions of type I interferons. Nat Rev Immunol 12: 125-135, 2012.

28. Kumagai Y, Takeuchi O, Kato H, Kumar H, Matsui K, Morii E, Aozasa K, Kawai T and Akira S: Alveolar macrophages are the primary interferon-alpha producer in pulmonary infection with RNA viruses. Immunity 27: 240-252, 2007.

29. Kim D, Martinez-Sobrido L, Choi C, Petroff N, García-Sastre A, Niewiesk $\mathrm{S}$ and Carsillo T: Induction of type I interferon secretion through recombinant Newcastle disease virus expressing measles virus hemagglutinin stimulates antibody secretion in the presence of maternal antibodies. J Virol 85: 200-207, 2011. 